\title{
Thermal Performance of a Pulsating Heat Pipe with Various Heating Conditions
}

\author{
Dong Soo Jang'1, Seonggi Park¹, Hanseok Mun', Junyub Lim¹, Yongchan Kim² \\ ${ }^{1}$ Graduate School of Mechanical Engineering, Korea University \\ Seoul 136-713, Republic of Korea \\ ${ }^{2}$ Department of Mechanical Engineering, Korea University \\ Seoul 136-713, Republic of Korea \\ nicebb0y@korea.ac.kr; jingjinge1@korea.ac.kr; hihansuk@gmail.com; diglim@korea.ac.kr; yongckim@korea.ac.kr
}

\section{Extended Abstract}

This study presents the heat transfer characteristics of a pulsating heat pipe for battery cooling of electric vehicles with various heating conditions. It is important to remove the heat from the electric vehicle battery and maintain uniform temperature on the battery cell for its stability and performance. Pulsating heat pipes have been studied extensively and used in various industries for their excellent thermal performance [1]. The pulsating heat pipe was made of copper tube with 8 turns. Two battery cell stack models were developed to simulate the performance according to direction of battery installations. One was arranged in vertical lines and the other was arranged in horizontal lines. The heating ratio between two battery cell stack models varied from 0.5 to 2 . The PHP worked in both vertical bottom heating mode and horizontal mode. FC-72 was used as a working fluid due to its fluid characteristics of low viscosity and surface tension. The filling ratio of the working fluid was $50 \%$. The filling ratio is defined as the volume of the working fluid divided by the total internal volume in the pulsating heat pipe. The effects of the battery cell stack arrangement, heating ratio, and inclination angle were analyzed at various heat inputs. The results showed that the thermal performance of the pulsating heat pipe was different according to working conditions. The battery cell stack arrangement, heating ratio, and inclination angle showed strong influence on the thermal characteristics of the pulsating heat pipe.

\section{References}

[1] X. Han, X. Wang, H. Zheng, X. Xu, G. Chen, "Review of the development of pulsating heat pipe for heat dissipation," Renew. Sustain. Energy Rev,. vol. 59, pp. 692-709, 2016. 